\title{
Expressão de cio de ovelhas Lacaune sincronizadas fora da estação de acasalamento
}

\author{
Expression of estrus of Lacaune sheep synchronized outside the breeding season \\ Expresión de celo de ovejas Lacaune sincronizadas fuera de la temporada de cria
}

Recebido: 28/03/2021 | Revisado: 02/04/2021 | Aceito: 25/11/2021 | Publicado: 06/12/2021

\author{
Karoline Barcellos da Rosa \\ ORCID: https://orcid.org/0000-0002-4890-4696 \\ Universidade Estadual do Rio Grande do Sul, Brasil \\ E-mail: karoline-rosa@uergs.edu.br \\ Rodrigo Flores Escobar \\ ORCID: https://orcid.org/0000-0003-1548-512X \\ Universidade Estadual do Rio Grande do Sul, Brasil \\ E-mail: rodrigo-escobar@uergs.edu.br \\ Rivas Matheus Lencina dos Santos \\ ORCID: https://orcid.org/0000-0001-9323-9149 \\ Universidade Estadual do Rio Grande do Sul, Brasil \\ E-mail: rivas-santos@uergs.edu.br \\ Tayã de Menezes Furtado Guedes \\ ORCID: https://orcid.org/0000-0001-7681-843X \\ Universidade Estadual do Rio Grande do Sul, Brasil \\ E-mail: tayamfguedes@hotmail.com \\ Gianny de Mello Maydana \\ ORCID: https://orcid.org/0000-0001-6237-3695 \\ Universidade Estadual do Rio Grande do Sul, Brasil \\ E-mail: gianny-maydana@uergs.edu.br \\ Thaís Moreira Osório \\ ORCID: https://orcid.org/0000-0003-3172-2412 \\ Universidade Federal do Pampa, Brasil \\ E-mail: thais-osorio@uergs.edu.br \\ Leonardo de Melo Menezes \\ ORCID: https://orcid.org/0000-0001-8536-0803 \\ Universidade Estadual do Rio Grande do Sul, Brasil \\ E-mail: leonardo-menezes@uergs.edu.br
}

\begin{abstract}
Resumo
O presente estudo foi realizado entre setembro e outubro de 2020, em Santana do Livramento, Rio Grande do Sul. O experimento objetivou avaliar a atividade reprodutiva de ovelhas leiteiras fora da estação convencional de acasalamento. Foram utilizadas 17 ovelhas multíparas da raça Lacaune. O protocolo hormonal utilizado consistiu no implante intravaginal por um período de sete dias do hormônio progesterona, somado a injeção intramuscular de Gonadotrofina coriônica equina (eCG) no momento da retirada do implante, na dose de 400 UI. Após o tratamento os animais foram submetidos a um período de encarneiramento por 5 dias. Registrou-se o momento do cio com auxílio de carneiros marcados com tinta no peito. As ovelhas apresentaram 52,9\% (9/17) de taxa de expressão de cio. Os resultados obtidos demonstram que o tratamento hormonal em ovelhas leiteiras possibilita a manifestação de cio em períodos do ano em que naturalmente não ocorreria.
\end{abstract}

Palavras-chave: Hormônios; Leite ovino; Progesterona; Reprodução.

\begin{abstract}
The present study was carried out between September and October 2020, in Santana do Livramento, Rio Grande do Sul. The experiment aimed to evaluate the reproductive activity of dairy sheep outside the conventional breeding season. Seventeen multiparous Lacaune ewes were used. The hormonal protocol used consisted of the intravaginal implant for a period of seven days of the progesterone hormone, added to the intramuscular injection of equine chorionic Gonadotropin (eCG) at the time of implant removal, at a dose of 400 IU. After treatment the animals were subjected to a period of mating for 5 days. The estrus was recorded with the help of sheep marked with ink on the chest. The sheep presented $52.9 \%$ (9/17) of estrus expression rate. The results obtained demonstrate that the hormonal treatment in dairy sheep allows the manifestation of estrus in periods of the year when it would not naturally occur.
\end{abstract}

Keywords: Hormones; Progesterone; Reproduction; Sheep milk.

\section{Resumen}

El presente estudio se realizó entre septiembre y octubre de 2020, en Santana do Livramento, Rio Grande do Sul. El experimento tuvo como objetivo evaluar la actividad reproductiva de las ovejas lecheras fuera de la temporada de cría convencional. Se utilizaron 17 ovejas Lacaune multíparas. El protocolo hormonal utilizado consistió en el implante 
intravaginal por un período de siete días de la hormona progesterona, más la inyección intramuscular de Gonadotropina Coriónica Equina (eCG) al momento de la extracción del implante, a una dosis de 400 UI. Después del tratamiento, los animales se sometieron a un período de encarnación durante 5 días. El calor se registró con la ayuda de ovejas marcadas con tinta en el pecho. Las ovejas presentaron 52,9\% (9/17) de tasa de expresión de estro. Los resultados obtenidos demuestran que el tratamiento hormonal en ovinos lecheros permite la manifestación del celo en épocas del año en que no ocurriría de forma natural.

Palabras clave: Hormonas; Leche de oveja; Progesterona; Reproducción.

\section{Introdução}

A ovinocultura representa um importante segmento dentro do setor pecuário, gerando divisas para o agronegócio brasileiro. A produção de lã, e mais atualmente carne, ocupam a grande maioria dos criatórios destinados a produção de ovinos no país. Existe ainda, a possibilidade de um terceiro produto, o leite, visando a agregação de valor através da produção de queijos finos. A ovinocultura leiteira, atividade bem estruturada em países europeus e asiáticos, é um tanto recente no Brasil. Alguns estudos recentes apontam para esta possibilidade na região da Fronteira Oeste do Rio Grande do Sul (Fontoura et al, 2020; Menezes et al, 2021). Com isso, a produção leiteira pode ser uma opção viável, apresentando-se como uma alternativa para produtores que buscam diversificar ou mesmo modificar seus modelos produtivos.

As Regiões Sul e Sudeste possuem maior produção e demanda do mercado consumidor para os produtos derivados do leite. A produção de leite exige cuidados nutricionais e sanitários diferenciados, e este novo modelo produtivo deve adaptar-se a esta condição. Animais leiteiros tendem a ter sua demanda energética ampliada, podendo-se incrementar o nível nutricional através de distintas estratégias, como pastejo horário em pastagens cultivadas (Munhoz et al, 2020), utilização de pastagens em período integral (Menezes et al, 2017) ou ainda em sistemas mais intensivos, baseados em suplementação com alimentos concentrados (Fontoura et al, 2020; Hernández et al, 2020; Silva et al, 2021; Menezes et al, 2021).

Neste sentido, destacam-se os ovinos da raça Lacaune, de origem francesa, introduzidas no Brasil no início da década de 90, sendo o estado do Rio Grande do Sul o primeiro a receber esses animais, que apresentam boa adaptação às condições de clima e alimentação no Sul do país (Brito et al., 2006; Senar, 2019). Quanto à produção leiteira, esta raça apresenta características interessantes aos produtores que investem nesta atividade: maiores teores de proteína e gordura no leite, resistência à mastite, a morfologia da mama e a facilidade de ordenha (Thomas et al., 2004; Coser et al., 2012).

Além da peculiaridade relacionada aos aspetos sanitários e nutricionais, existe ainda a questão logística de produção de leite ovino: a necessidade de diversificar a época de partos no rebanho, a fim de permitir grupos de partos em distintos períodos, para manutenção da produção de leite durante o ano todo. Portanto, o período de reprodução é fundamental para qualquer sistema de produção, considerando sua influência direta nos indicadores de produtividade do rebanho, como na taxa de ovulação, taxa de concepção e número de crias nascidas (Maia \& Nogueira, 2019).

Neste contexto, a reprodução tem papel fundamental no sucesso de um empreendimento dedicado a atividade leiteira. De maneira geral, os animais apresentam dois tipos de classificação de acordo com o fotoperíodo: animais de dias longos, estando inseridos os equídeos e os bovinos, uma vez que a atividade sexual se manifesta após o solstício de inverno, quando os dias são mais longos; e os animais de dias curtos, no qual estão inclusos os ovinos, caprinos e suínos, os quais a atividade sexual se manifesta após o solstício de verão, quando os dias são mais curtos. Portanto, o fotoperíodo é um dos principais fatores ambientais determinantes da estacionalidade reprodutiva das fêmeas ovinas (Traldi, 2002).

Desta forma, haverá dentro de um ano a época convencional de acasalamento (fim de verão e meses de outono) e época em que é necessário lançar mão de métodos alternativos de indução da ovulação. Neste sentido, há uma crescente demanda por biotécnicas de reprodução em pequenos ruminantes que visem o incremento da produtividade e da rentabilidade dos rebanhos e das unidades reprodutivas (Gusmão \& Andrade, 2005). Conforme Carvalho (2009), a sincronização do estro e da ovulação trazem reais benefícios, como melhoria da eficiência reprodutiva, redução da mão de obra para detecção do estro, 
concentração das parições, lotes mais homogêneos ao abate e melhor utilização do reprodutor. Além de possibilitar altas taxas de prenhez no início das estações de monta, diminuição no intervalo entre partos e aumento no número de animais nascidos, permitindo maiores cuidados com os cordeiros e incremento da distribuição da oferta de carne ao longo do ano. Com isso, biotecnologias que permitam a otimização dos processos reprodutivos tendem a gerar retornos diretos e indiretos para os produtores e técnicos, uma vez que irão viabilizar a redução do intervalo entre partos, altas taxas de prenhez e nascimentos sincronizados, facilitando, inclusive, o manejo nutricional destes animais. Assim, o objetivo deste trabalho foi avaliar a atividade reprodutiva de ovelhas Lacaune expostas a um protocolo de sincronização de cio.

\section{Materiais e Métodos}

Esta pesquisa desenvolveu-se de forma exploratória, consistindo na descrição do comportamento e taxa de cios mediante o protocolo adotado, sendo o mesmo elaborado conforme Pereira et al. (2018).

O estudo foi realizado no município de Santana do Livramento-RS, situado na região da Fronteira Oeste do estado do Rio Grande do Sul, coordenadas $30^{\circ} 48^{\prime} 41^{\prime \prime S}$ e $55^{\circ} 28^{\prime} 05^{\prime \prime} \mathrm{W}$. A propriedade localiza-se na região do munícipio denominada Cerro da Vigia, tendo como atividades a vitivinicultura, olericultura, suinocultura e criação de ovelhas leiteiras. O experimento foi conduzido entre os meses de setembro e outubro de 2020, fora da estação reprodutiva convencional. O rebanho de ovinos leiteiros consiste em 120 animais, pertencentes às raças Lacaune, Crioulas e suas cruzas, mantidas em regime semi-intensivo de criação. Os animais foram manejados sob pastagens nativas e cultivadas de azevém (Lolium multiflorum) e aveia (Avena sativa) e recebiam alimentação à base de feno de alfafa, silagem de milho, sal mineral e ração correspondente a 1,0\% de peso vivo, durante o período de lactação.

As coletas de dados ocorreram entre os meses de setembro e outubro de 2020. Foram utilizados no experimento 17 ovelhas da raça Lacaune, identificadas com brincos numerados. Antes do início do tratamento, foi realizado o exame clinico para verificar o estado nutricional e de saúde das matrizes. Além da avaliação geral, utilizou-se o método de escore de condição corporal (ECC). As fêmeas apresentaram no início do experimento condição corporal mínima de 2,5com variação entre 2,5 a 3,5. Além disso, verificou-se o histórico individual de cada matriz, utilizando-se apenas matrizes já paridas em um período mínimo de 90 dias.

O protocolo utilizado nas 17 fêmeas da raça Lacaune contou com a introdução de um implante intravaginal contendo o hormônio acetato de medroxiprogesterona (MAP), que permaneceu no interior da vagina por um período de sete dias. Após este período, no momento da retirada aplicou-se em cada ovelha Gonadotrofina Coriônica Equina (eCG), por via intramuscular profunda, na dose de 400 UI. A introdução da esponja com 60 mg de MAP (foi realizada com o auxílio de um aplicador de PVC, com tamanho compatível com as fêmeas. O aplicador foi higienizado e mergulhado em um recipiente contendo desinfetante à base de iodo (Biofor). A esponja recebeu antibiótico injetável (1 $\mathrm{mL}$ de oxitetraciclina) para diminuir a intensidade de possíveis vaginites e foi fixada dentro do aplicador de PVC. A retirada da esponja intravaginal das matrizes ocorreu sete dias após a introdução da mesma. Após a retirada do implante realizou-se a aplicação de 400 UI de eCG intramuscular, com a finalidade de promover o crescimento final e luteinização do folículo.

Após este tratamento os animais foram submetidos a exposição por carneiros contendo buçal marcador por cinco dias. Desta forma, possibilitou-se a marcação da garupa das fêmeas, permitindo-se o registro do momento da expressão de cio.

\section{Resultados e Discussão}

Observou-se a taxa de expressão de cio de 52,9\% (9/17). Estes resultados são semelhantes aos obtidos por Maxwell \& Barnes (1986), que utilizando protocolos de longa duração, verificaram manifestação de cio em aproximadamente metade das fêmeas tratadas com progestágeno, mantido durante 12 a 14 dias, em associação com ECG, sendo que o estro ocorreu em 
média 36 h após o término do tratamento. Madeira et al (2020) também encontraram resultados equivalentes (54\% de expressão de cio), utilizando protocolo de curta duração (7 dias) sem utilização de ECG. Os dados referentes ao momento da expressão de cio podem ser observados na Tabela 1.

Tabela 1 - Manifestação do estro (em horas) após a remoção do implante de progestágeno em fêmeas ovinas (em porcentagem).

\begin{tabular}{cc}
\hline Manifestação do estro (horas) & Fêmeas (\%) \\
\hline$>12 \mathrm{~h}$ & - \\
$12-24 \mathrm{~h}$ & 44,4 \\
$24-36 \mathrm{~h}$ & 33,3 \\
$36-48 \mathrm{~h}$ & - \\
$48-60 \mathrm{~h}$ & 22,2 \\
$60-72 \mathrm{~h}$ & - \\
$72-84 \mathrm{~h}$ & - \\
$84-96 \mathrm{~h}$ & - \\
$96-108 \mathrm{~h}$ & - \\
$108-120 \mathrm{~h}$ & \\
\hline
\end{tabular}

Fonte: Autores (2021).

Dentre as ovelhas que manifestaram estro após a utilização do protocolo, 44,4\% (4/9) das fêmeas demonstraram cio entre $12 \mathrm{~h}$ e 24 h após a retirada do implante; 33,3\% (3/9) entre 24-36 h, e 22,2\% (2/9) entre 48-60 h. Não houve verificação de estro após este período. Estes dados são similares aos de Castilho et al. (2007), que avaliando protocolos com progestágeno, prostaglandina e ECG, observaram sinais de estro entre 24-48 h após a remoção do implante de progesterona, corroborando os dados do presente experimento. Ungerfeld e Rubianes (2002) obtiveram 95\% da manifestação de estro até 40 horas após a retirada do dispositivo, trabalhando com um protocolo similar (6 dias de progesterona e 380 UI de ECG). Da mesma forma, Santos e Barcelos (2012), estudando eficiência de dois protocolos de sincronização do ciclo estral em ovinos no momento da manifestação de estro encontraram $75 \%$ das ovelhas manifestando o estro em até 72 h após a retirada do implante.

\section{Considerações Finais}

O protocolo contendo progestágeno e eCG apresentou resultado satisfatório, condizente com os dados encontrados na literatura.

A maior concentração de estros dá-se no período até 36 horas após retirada dos implantes. Neste sentido, comportamento do cio segue um padrão semelhante a ovelhas de outras raças sincronizadas fora da estação reprodutiva.

\section{Agradecimentos}

Os autores agradecem o auxílio financeiro da FAPERGS e UERGS, através das bolsas de pesquisa. Ainda, à Cabanha Terroir da Vigia, pela cedência do material experimental.

\section{Referências}

Brito, M. A., Diaz González, F., Ribeiro, L. A., Campos, R., Lacerda, L., Barbosa, P. R. \& Bergmann, G. 2006. Composição do sangue e do leite em ovinos leiteiros do sul do Brasil: variações na gestação e na lactação. Ciência Rural, 36(3), 942-948.

Carvalho, J. A. Ovelhas da raça Santa Inês após tratamento com progestágeno novo e reutilizado associado a ecg ou fshp. 2009. 566 . Dissertação de mestrado - Curso de PósGraduação em Zootecnia, Universidade Estadual do Sudoeste da Bahia - UESB / Campus de Itapetinga - BA.

Castilho, C., Mori, M. M., Alessi, C. P. \& Giuffrida, R. 2007. Indução de estro em ovelhas da raça Texel durante o anestro estacional utilizando meio implante de progestágeno novo ou reutilizado. Vet Not, 13, 39-45. http://www.seer.ufu.br/index.php/vetnot/article/view/18832 
Coser, S. M., Lopes, M. A. \& Costa, G. M. 2012. Mastite bovina: controle e prevenção. Universidade Federal de Lavras, Lavras-MG. In: Boletim Técnico (93), 1-30.

Fontoura, E. A. B, Tâmara, J. Q., Rodrigues, D. P., Maydana, G. M., Santos, R. M. L., Munhoz, M. L., Corrêa, G. F. \& Menezes, L. 2020. Características da lactação de ovelhas Texel criadas extensivamente. Brazilian Journal of Development., 6(1), 1586-1597. https://doi.org/10.34117/bjdv6n1-109

Gusmão, A. L. \& Andrade, J. M. M. 2005. Transferência de embriões em caprinos e ovinos. Acta Scientiae Veterinae. 33(Supl.1), 29-33. https://seer.ufrgs.br/ActaScientiaeVeterinariae/article/view/14418/8348

Hernandez, J. C. A., Alberto, R. V., KebreaB,E., AppuhamY, J. A. D. R. N., Dougherty, H. C., Ortega, O. C. \& Ronquillo, M. G. 2020. Effect of forage to concentrate ratio and fat supplementation on milk composition in dairy sheep: A meta-analysis,Livestock Science, $238,104069$. https://doi.org/10.1016/j.livsci.2020.104069

Madeira, M., Dos Santos Araújo, K., Vieira Dos Santos, L., Machado Montezano, R. \& Ferreira Corrêa, G. Eficácia De Diferentes Protocolos Reprodutivos Em Ovelhas Da Raça Texel. Anais do Salão Internacional de Ensino, Pesquisa e Extensão, 10(2). https://guri.unipampa.edu.br/uploads/evt/arq_trabalhos/17673/seer_17673.pdf

Maia, M. S. \& Nogueira, D. M. Manejo reprodutivo de caprinos e ovinos em regiões tropicais. Petrolina, PE: Embrapa Semiárido, 2019.

Maxwell, W.M. C. \& Barnes, D. R. 1986. Induction of estrus in ewes using a controlled internal drug release device and PMSG. J Agric Sci, v.106, p.201-203. https://doi.org/10,1017/S0021859600061931

Menezes, L. M., Fernandes, M. V. B. \& Silva, I. M. 2021. Efficiency of creep feeding on the performance of Ideal sheeps and Merino Australiano x Ideal lambs. Research, Society and Development, [S. l.], v. 10, n. 2, p. e34110212663. https://doi.org/10.33448/rsd-v10i2.12663.

Menezes, L. M., Fontoura, E. A. B., Damilano, A. S., Rosa, R. S., Perez, H. A., Gomes, A. F. F., Cunha, P. T., Chagas, R. A. \& Corrêa, G. F. 2017. Desempenho de cordeiros Texel e Corriedale mantidos em azevém em fim de ciclo. Revista electrónica de Veterinaria. 18(12):1-9. https://doi.org/10.34117/bjdv6n1-353

Menezes, L. de M.., Fontoura, E. A. B. da., Tâmara, J. Q.., Munhoz, M. da L., Rodrigues, D. P., \& Gomes, A. F. F. 2021. Milk yeld of Texel sheeps submitted to suplementation. Research, Society and Development, 10(2), e30010212500. https:// doi.org/10.33448/rsd-v10i2.12500

Munhoz, M. L., Fontoura, E. A. B., Rodrigues, D. P., Moreira, C. H., Rodrigues, P. E. B., Cordeiro, D. O., Santana, G. A. O. \& Menezes, L. M. 2020. Desempenho de ovelhas e cordeiros Texel em distintas fases do manejo nutricional. Brazilian Journal of Development, 6, $4909-4919$. https://doi.org/10.34117/bjdv6n1-353

Pereira A. S., Shitsuka, D. M., Pereira, F. J. \& Shitsuka, R. (2018). Metodologia da pesquisa científica. UFSM. https://repositorio.ufsm.br/bitstream/handle/1/15824/Lic_Computacao_Metodologia-Pesquisa-Cientifica.pdf?sequence=1

Santos, F. C. C. \& Barcelos, R. A. D. 2012. Eficiência de protocolos de sincronização de estro em ovelhas. Rev. bras. reprod. anim, 36(3), 202-205. http://www.cbra.org.br/pages/publicacoes/rbra/v36n3/p202-205\%20(RB399).pdf

Senar. 2019. Serviço Nacional de Aprendizagem Rural. Ovinocultura: criação e manejo de ovinos de leite / Serviço Nacional de Aprendizagem Rural. Brasília: https://www.cnabrasil.org.br/assets/arquivos/264-Ovinocultura-cria\%C3\%A7\%C3\%A3o-e-manejo-de-ovinos-de-leite_191025_203520.pdf

Silva, I. M, Fernandes, M.V. B \& Menezes, L. M. 2021. Avaliação de um sistema de ovinocultura intensificada baseado em campo nativo no Rio Grande do Sul. Conjecturas, 21(5), 671-680. https://doi.org/10.53660/conj-343-512

Thomas, D. L., Berger, Y. M., Gottfredson, R. G. \& Taylor, T. A. Comparison of East Friesian and Lacaune breeds for dairy sheep production in North America. Proc. 10th Great lakes Dairy Sheep Symp. Hudson, Wisconsin: University of Wisconsin-Madison, Dept. of Anim. Sci, 115-123, 2004. https://citeseerx.ist.psu.edu/viewdoc/download?doi=10.1.1.573.6465\&rep=rep1\&type=pdf

Traldi, A.S. Utilização da biotecnologia na otimização do manejo reprodutivo de ovinos. In: II Simpósio Mineiro de Ovinocultura. Lavras-MG: UFLA. Anais.... Lavras-MG. 167-185, https://edisciplinas.usp.br/pluginfile.php/4937797/mod_resource/content/1/manejo\%20reprodutivo\%20de\%20ovinosatualizado.pdf

Ungerfeld, R. \& Rubianes, E. 2002. Short term primings with different progestogen intravaginal devices (MAP, FGA and CIDR) for eCG-estrous induction in anestrus ewes. Small Ruminant Research, 46(1), 63-66. https://doi.org/10.1016/S0921-4488(02)00105-0 